\title{
A Rare Case of Pneumococcal Appendicitis in a Child
}

\author{
Amar Chikhaoui $\mathbb{D}^{1},{ }^{1}$ Néhémie Nzoyikorera, ${ }^{1,2}$ Mehdi El Mouadden, ${ }^{3}$ \\ Mounia Al Zemmouri, ${ }^{1,3}$ and Khalid Zerouali ${ }^{1,2}$ \\ ${ }^{1}$ Faculty of Medicine and Pharmacy of Casablanca, Hassan II University of Casablanca, Casablanca, Morocco
${ }^{2}$ Bacteriology, Virology and Hospital Hygiene Laboratory, Ibn Rochd University Hospital, Casablanca, Morocco
${ }^{3}$ Pediatric Visceral Surgery, Ibn Rochd University Children's Hospital, Casablanca, Morocco
}

Correspondence should be addressed to Amar Chikhaoui; amar.chikhaoui-etu@etu.univh2c.ma

Received 21 June 2021; Revised 25 December 2021; Accepted 20 January 2022; Published 7 February 2022

Academic Editor: Maria Moschovi

Copyright (c) 2022 Amar Chikhaoui et al. This is an open access article distributed under the Creative Commons Attribution License, which permits unrestricted use, distribution, and reproduction in any medium, provided the original work is properly cited.

\begin{abstract}
Appendicitis is the most common cause for abdominal surgery in children. It is usually caused by Escherichia coli and Streptococcus species and is generally polymicrobial. However, Streptococcus pneumoniae is a rare cause of appendicitis. We report a rare case of pneumococcal appendicitis in a 7-year-old child with no underlying conditions, in association with $E$. coli and group $\mathrm{F} \beta$-hemolytic Streptococcus. The isolated pneumococcal strain was sensible to all tested antibiotics. The patient had a full recovery after surgery and antibiotics. This case emphasizes that $S$. pneumoniae can cause a variety of unusual infections like appendicitis, in patients with or without underlying conditions. Thus, even though being a rare entity, physicians should always be aware of S. pneumoniae as a possible causative agent.
\end{abstract}

\section{Introduction}

Streptococcus pneumoniae (S. pneumoniae) can cause a wide spectrum of infections like meningitis, pneumonia, bacteremia, and otitis. Furthermore, it has been reported that the pneumococcus can demonstrate very atypical presentations, like cardiovascular, genitourinary tract, and gastrointestinal infections [1]. We report a rare case of $S$. pneumoniae causing appendicitis in a child.

\section{Case Presentation}

A 7-year-old male child presented at Ibn Rochd University Children's Hospital with a history of abdominal pain localized in the right lumbar region, fever, and food emesis for two days. No intestinal transit disorders were reported. Abdominal examination showed right lower quadrant abdominal pain (positive McBurney sign) and no signs of peritoneal irritation. Rovsing, Blumberg, and Psoas signs were negative. The patient had a fever of $38.6^{\circ} \mathrm{C}$. There were no other pathological findings on physical examination.
Laboratory analysis highlighted a white blood cell count of $10.2 \times 10^{3} / \mathrm{mm}^{3}$ (83\% of neutrophils) and a C-reactive protein level of $272.8 \mathrm{mg} / \mathrm{L}$. No other biological or radiological exams were done as the patient was directly taken to the operating room. The final diagnosis was acute appendicitis.

The abdomen was opened by McBurney incision. An inflamed, enlarged, and perforated appendix with periappendicular pus was found perioperatively. The rest of the peritoneal cavity was clean. A pus sample was collected, and the appendicectomy was done. The pus was sent to the laboratory for bacteriological analysis.

Gram-stained direct smear showed Gram-positive cocci. The culture was polymicrobial with S. pneumoniae, E.coli, and group $\mathrm{F} \beta$-hemolytic streptococci. Antimicrobial sensitivity testing was done for $S$. pneumoniae following the EUCAST guidelines. The pneumococcal strain was sensitive to penicillin G, ceftriaxon, erythromycin, tetracycline, cotrimoxazol, and levofloxacin. Serogrouping/serotyping was done by the checkerboard method with Pneumotest-Latex (Statens Serum Institute antisera, Copenhagen, Denmark) 
and multiplex PCR according to the protocol proposed by the CDC [2], but the serotype has not been identified. Thus, the strain was categorized as a nonvaccine serotype (NVS).

After the procedure, the patient had a full recovery on a treatment consisting of an association of gentamycin $100 \mathrm{mg}$ IV daily for three days, ampicillin-sulbactam $3 \mathrm{~g}$ IV daily, and metronidazole $1.2 \mathrm{~g}$ IV daily for five days. Oral antibiotic treatment was continued 5 days after discharge.

\section{Discussion}

Intra-abdominal infections and particularly appendicitis are rarely caused by $S$. pneumoniae. The estimated incidence rate of pneumococcal appendicitis is $0.25 / 100000$ [3]; Heltberg et al. reported only six cases of pneumococcal appendicitis from 1967 to 1981 in 2 hospital settings, representing approximately $0.3 \%$ of appendicitis patients [4].

The etiology of appendicitis is usually polymicrobial and associated with common intestinal pathogens, which corresponds to our case. However, in most reported cases of pneumococcal appendicitis, $S$. pneumoniae was detected in pure cultures and that make our case a rare occurrence [5-8].

Some hypotheses have been considered to explain the involvement of $S$. pneumoniae in appendicitis. The colonization of the bowel may be the source of the pathologic process, but $S$. pneumoniae is very susceptible to the bactericidal effect of gastric acid. Furthermore, no studies have established the sporadic presence of the pneumococcus in the intestinal flora. The appendix could also be reached by hematogenous spread from the colonized respiratory tract, particularly in children, whose nasopharynxes are densely colonized. It should also be noted that a number of proved appendicitis patients had a history of respiratory symptoms of pneumonia [9].

Few articles report microbiological characteristics in case reports. As in ours, the majority reported $S$. pneumoniae strains that were sensitive to most tested antibiotics $[5,6,8]$. In Casablanca, vaccination has considerably reduced the prevalence of multiresistant serotypes, which were often associated with some of the prevaccination serotypes covered by PCV, such as 9V, 6B, 14, 19A, 19F, and 23F [10]. However, only a handful reported the serotypes of the isolated strains: serogroup 19 was the most prevalent followed by serotype 3 [5]. Concerning our case, a NVS was found.

The most frequent risk factors associated with invasive pneumococcal disease include patients with cardiopathy, chronic lung disease, diabetes, immunosuppression (nephrotic syndrome, malignancies, etc.), and splenic dysfunction (splenectomy, asplenia, etc.) [11]. However, in our case study, none of these factors were present and the vaccination status was unknown. Male gender could be the only risk factor in our case, which is documented in the literature [12]. This concords with findings from other case reports where most patients had no predisposing factors for pneumococcal infections $[4,5]$.

According to the World Journal of Emergency Surgery guidelines, triple agent antibiotic therapy should be administered after appendicectomy in cases of perforated appendicitis in children. Several options are possible, one of which is the association of ampicillin-metronidazole and gentamycin, which was used in our case. Alternatives include ceftriaxone-metronidazole or ticarcillin-clavulanate plus gentamicin, in accordance with the epidemiology of bacteria [13]. The duration of the treatment ought to be shorter than 7 days, and transition to oral therapy should be done as soon as possible, since oral antibiotics show equivalent outcomes compared with intravenous therapy, but with shorter length of hospitalizations [13].

In our case report, the patient had a full recovery with no complications. This concords with reported cases like the case of Ghadage [6]. Heltberg et al. reported 2 patients who presented mild complications while Dimond and Proctor reported a particular case of a 9-year-old patient who presented pneumococcal meningitis postoperatively from a peritonitis secondary to an appendicitis $[5,14]$.

\section{Conclusion}

We reported a rare case of pneumococcal appendicitis in a child without any predisposing factors. A positive outcome was observed after surgery and antibiotic therapy. Although uncommon, S. pneumoniae can also cause appendicitis and thus should be considered as a probable cause by physicians in abdominal infections.

\section{Data Availability}

The data used in this article can be found in the archives of the Pediatric Visceral Surgery Department of Ibn Rochd University Hospital.

\section{Conflicts of Interest}

The authors declare that there are no conflicts of interest regarding the publication of this paper.

\section{Authors' Contributions}

Study design was devised by KZ. Data collection and laboratory analysis were performed by AC and NN. Analyses were carried out by AC and NN. AC wrote the manuscript, and all authors contributed to revisions. All authors read and approved the final manuscript.

\section{References}

[1] S. N. Taylor and C. V. Sanders, "Unusual manifestations of invasive pneumococcal infection," The American Journal of Medicine, vol. 107, no. 1, pp. 12-27, 1999.

[2] inMeningitis | Lab Manual | PCR Detection and CharacterizationCDC, New York, NY, USA, 2019.

[3] P. Gérôme, N. Bourilhon, B. Soullie, B. Foucher, M.-P. Otto, and F. Milou, "Acute appendicitis due to both Klebsiella pneumoniae and serotype 35B Streptococcus pneumoniae, an emergent serotype," Annales de biologie clinique, vol. 69, no. 4, pp. 485-488, 2011.

[4] O. Heltberg, B. Korner, and P. Schouenborg, "Six cases of acute appendicitis with secondary peritonitis caused 
byStreptococcus pneumoniae," European Journal of Clinical Microbiology, vol. 3, no. 2, pp. 141-143, 1984.

[5] J. Caierão, A. F. Cornely, G. R. da Cunha, M. Mott, A. P. Zavascki, and C. Dias, "Streptococcus pneumoniae appendicitis in an adult patient," The American Journal of Emergency Medicine, vol. 33, no. 7, pp. 990-993, 2015.

[6] D. P. Ghadage, "Appendicitis in a child due to Streptococcus pneumoniae: a rare case report," Journal of Clinical and Diagnostic Research, vol. 9, 2015.

[7] X. Argemi, N. Lefebvre, M. Baldeyrou, N. Douiri, and Y. Hansmann, "Streptococcus pneumoniae appendicitis and bacteremia in an immunocompetent adult," Medecine et Maladies Infectieuses, vol. 46, no. 2, pp. 106-107, 2016.

[8] A. Govindaswamy, S. Sagar, V. Trikha, S. Mittal, R. Malhotra, and P. Mathur, "Atypical case presentations of Streptococcus pneumoniae from level 1 trauma centre in India-A case series," Journal of Family Medicine and Primary Care, vol. 9, no. 9, pp. 5058-5061, 2020.

[9] D. Miron, I. Dashkovsky, M. Zuker, S. Szvalb, and C. Cozacov, "Primary streptococcus pneumoniae appendicitis in a child: case report and review," The Pediatric Infectious Disease Journal, vol. 22, no. 3, pp. 282-284, 2003.

[10] I. Diawara, K. Zerouali, K. Katfy et al., "Invasive pneumococcal disease among children younger than 5 years of age before and after introduction of pneumococcal conjugate vaccine in Casablanca, Morocco," International Journal of Infectious Diseases, vol. 40, pp. 95-101, 2015.

[11] C. Levy, E. Varon, N. Ouldali, S. Béchet, S. Bonacorsi, and R. Cohen, "Changes in invasive pneumococcal disease spectrum after 13-valent pneumococcal conjugate vaccine implementation," Clinical Infectious Diseases, vol. 70, p. ciz221, 2019.

[12] T. Q. Tan, "Pediatric invasive pneumococcal disease in the United States in the era of pneumococcal conjugate vaccines," Clinical Microbiology Reviews, vol. 25, no. 3, pp. 409-419, 2012.

[13] S. Di Saverio, M. Podda, B. De Simone et al., "Diagnosis and treatment of acute appendicitis: 2020 update of the WSES Jerusalem guidelines," World Journal of Emergency Surgery, vol. 15, no. 1, p. 27, 2020.

[14] M. Dimond, "Concomitant pneumococcal appendicitis, peritonitis, and meningitis," Archives of Surgery, vol. 111, no. 8, p. 888, 1976. 